\title{
Investigation on Flower Consumption and Development Strategy of Guangzhou
}

\author{
Wu Xin ${ }^{1}$, Ling Qingyan², Zhong Sisi ${ }^{1}$ \\ ${ }^{1}$ Guangzhou Nanyang Polytechnic, Guangzhou, Guangdong, 510925, China \\ ${ }^{2}$ Nanchang Institute of Technology, Nanchang, Jiangxi, 330000, China
}

Keywords: Guangzhou, questionnaire surveys, flower consumption, flower industry

\begin{abstract}
Based on the first-hand data obtained from a large number of random questionnaire surveys, this paper makes an in-depth analysis of the current status of Guangzhou residents' flower consumption, consumption characteristics and influencing factors of consumption. The results show that the main factors affecting the consumption of flowers include the age of the consumers, the level of education received, the monthly average income, the monthly living expenditure level, and the proportion of enjoying consumption. At the same time, other factors such as flower varieties, network resources, flower culture, and government policies also play a very important role. Finally, according to the conclusions of the study, the countermeasures and suggestions for promoting the flower consumption of Guangzhou residents are proposed, which will have important practical significance for driving the development of the flower industry in Guangzhou.
\end{abstract}

\section{Introduction}

The flower industry is a new green industry with economic, social and ecological benefits, as well as an intensive sunrise industry integrating labor, capital and technology ${ }^{[1]}$. The development of flower industry plays an important role in promoting economic and social development and the construction of ecological civilization. At the same time, it can accelerate the adjustment of industrial structure, increase the income of farmers and improve people's life quality ${ }^{[2]}$. In $21^{\text {st }}$ century, the scale of Chinese flower industry develops steadily, production pattern has been basically formed, and foreign cooperation is expanding, and a more complete flower industry chain has been gradually formed ${ }^{[3]}$. Under the development background of global economic integration and "The Belt and Road", the flower industry in China is facing new challenges.

With the forefront flower planting scale and sales output value, Guangzhou is the most important flower distributing center in our country. As a transit station for flower transportation in Southern China and Pearl River Delta region, Guangzhou has bred a large number of flower wholesale trade groups and flower wholesale matching markets. The flower wholesale market of Lingnan Guangzhou is the largest trading market of fresh flower as well as cut leaf and branch in China. The Southern China flower wholesale market is a high-end flower trading center. The matching market of Guangzhou flower expo garden is a comprehensive market with relatively perfect supporting facilities and marketing mode, as well as an industrial center in which flowers are produced, supplied and sold ${ }^{[4]}$.

Due to regional economic development of the "Silk Road on the Sea in $21^{\text {st }}$ Century" and proposition of "The Belt and Road", flower industry in Guangzhou will be further integrated into the "The Belt and Road" strategy with advantages of resources, policy and location. The interconnection and trade in goods brought by "The Belt and Road" strategy make the transportation and export of flowers towards the world more smooth and convenient. At the same time, the export of Guangzhou flowers is faced with some serious problems, such as the lack of independent intellectual property right of fine flower varieties, low scientific and technological elements of flower breeding, backward flower production management method, deficient network of storage, transportation and marketing and so on ${ }^{[5-10]}$. Flower industry in Guangzhou is a microcosm of China's flower industry, being in the state of opportunities and challenges. 


\section{Investigation Methods and Data}

In this survey, we conducted a random survey of consumers and shopkeepers in the market during March to June in 2017 in the way of questionnaire and interview. According to the proportion of population in each district of Guangzhou Central District, the number of people sampled in each district was estimated. Taking into account the randomness of questionnaire, several representative places were selected. These were Zheng Jia Square in Tianhe District, Beijing Road Pedestrian Street in Yuexiu District, Shang Xia Jiu Pedestrian Street in Liwan District, Liying Square in Haizhuqu District and Baixin Square in Baiyun District. Besides, 500 samples were collected for questionnaire inquiry by simple sampling survey. Finally, a total of 480 valid questionnaires were collected, with an effective recovery rate of $96 \%$. The questionnaire is based on a large number of literature collection and data analysis, dividing into two parts: the first part is the basic information of respondents, including gender, age, educational level, average monthly income etc, and the second part is the flower consumption of respondents, mainly covering buying time of all kinds of flower, flower types respondents prefer to purchase and so on.

\section{Results and Analysis}

Among respondents, those who support that flower price in the flower market is high but acceptable amount to $78.2 \%$. The proportion who believes that the price is high and unacceptable is $4.6 \%$ and $17.2 \%$ consumers are not sure whether the present price is high or low. This shows that the price evaluation of most Guangzhou residents is acceptable, the increase in prices will not affect the purchase quantity and the demand for flowers relatively lacks price elasticity.

Most residents in Guangzhou believe that they have "little flower knowledge", accounting for 32.4\%. 10.9\% hold that their flower knowledge is "comparative more", and 10.34\% think they have "basic flower knowledge". This demonstrates that, generally speaking, Guangzhou residents' knowledge about flower conservation is relatively lacking, which is also a key factor restricting their flower consumption.

Guangzhou residents have more ways to buy flowers. The results show that the first choice is "flower market", the percentage of which is $40.4 \%$. The followed one is "flower franchised shop", with a proportion of $16.1 \%$. People choosing "floating flower peddlers" and "farmers market" are similarly $24.7 \%$. Those choose "large supermarket" and "nursery center" are equal in number, amounting to 5.5\% respectively. There are $7.8 \%$ residents choosing online ordering, which are more than those who choose "large supermarket" and "nursery center".

There are respectively $41.7 \%$ and $20.5 \%$ Guangzhou residents obtaining flower information through "self seeking" and "relatives and friends", followed by "newspapers, magazines" and "Internet advertising". It can be seen that they are more active and enthusiastic in exploring flower information and searching for information by themselves or from friends and relatives. With the development of information technology, Internet has become the main channel to get information about merchandise purchase and sale.

The survey shows that the time spent by residents buying flowers is concentrated in three important festivals, namely Spring Festival, Valentine's Day and Mother's Day. They purchase most flowers during Spring Festival, amounting to 24.5\%. In addition to Spring Festival, other festivals of traditional Chinese traditional festivals include Tomb-sweeping Day, Tanabata Festival, Dragon Boat Festival and Chongyang Festival, during which residents' purchasing probability is lower than that in Western Valentine's Day and Mother's Day. The number of people buying flowers during non-festivals accounts for $7.6 \%$, ranking the fourth. This also manifests that the consumption behavior of Guangzhou residents is becoming more and more popular. They will buy flowers during non-festivals, no longer limited to specific days.

During Spring Festival, Guangzhou residents prefer to buy red flowers, such as peach blossom, poinsettia, rose, lily implying "deep blessings and good families" (such as perfume lily), moth orchid which has a good meaning of "full happiness and smooth career", orange meaning "good fortune" (such as Kumquat and Citrus oleocarpa Tanaka), and narcissus that mean "miss and 
reunion". All of these reflect that Guangzhou residents focus on the orientation of traditional culture in association with festival environment and the meaning of flower itself when buying flowers.

Guangzhou residents prefer to buy ornamental potted flower, orchid and lily flowers during non-festivals. Ornamental potted flowers are deeply loved by Guangzhou residents because of bright colors, peculiar flower shapes and fragrance. Orchid, such as moran, chlorophytum, dendrobium and phalaenopsis representing elegance, and lily meaning "beauty and deep blessing" are popular with the people of the city.

According to the survey results, there are differences in consumption behavior between different consumer groups. The author sets up basic information of consumers in the questionnaire, and analyzes the differences in flower consumption behavior of Guangzhou residents from five aspects, such as gender, age, occupation, education level and individual monthly income.

Because of different genders, there are obvious differences in the experience of buying flowers in the past one year. The proportion of men who did not buy flowers in 2016 was higher than that of women. Reasons for not buying of men were mainly reflected in "no need", "purchasing inconvenience" and "no maintenance". Women did not buy because of "higher price" as well as "flower quality problems". This shows that men generally do not want to invest too much time and energy in conserving flowers.

In terms of the types of flowers, the percentage of men buying potted plants and emulation flowers is higher than that of women, while the proportion of women buying fresh cut flowers and bonsai is significantly higher than that of men. It can be seen that men pay more attention to the persistence of flowers, while women stress the beautification of flowers and the effect of air purification. The main purpose of men to buy fresh cut flowers is to give people as gifts, but the purpose of women is for decoration.

When flowers are consumed, there are differences between consumers of different genders. Men generally pay more attention to the convenience of the purchase and visual effects, while women generally consider the price of and maintenance management.

The most favorite flower types for those under 16 years are fresh cut flowers, especially rose, chrysanthemum and carnation. 16-25 year old consumers prefer to buy fresh cut flowers and potted flowers. With the increase of age, the probability of buying potted flowers is increasing gradually, and the most favorite of old people aged above 56 is potted flowers and bonsai.

The relationship between ages and reasons for no purchase of flowers reflects that people who do not buy flowers are mostly those under 16 and the main reason is the high price and no self-sufficiency of economic capacity. Then is 36-45 and 46-55 year old consumers. Consumers above 56 years old had more flower purchasing experience than any other age group in 2016, indicating that this age group is mostly retirees with more energy and time, as well as the power of consumption. Moreover, the cultivation and maintenance of potted flowers are conducive to pleasing and nourishing hearts. Therefore, this group is the major potential consumers. Those at the age between 26 to 35 are located in the second place, who pay more attention to the creation of romantic atmosphere and the improvement of life quality, without heavy economic pressure.

In terms of the relationship between educational level and whether to buy flowers, the proportion of consumers graduated from junior colleges and undergraduate colleges buying flowers in the past year is relatively high. In addition, the probability of buying flowers varies with people's educational background, while the percentage of consumers with master's and above education reduced because of "no time and energy".

In the type of flowers, students from junior high school or below grades, high school and secondary school prefer to buy fresh cut flowers. However, as their educational background increases, they tend to buy flowers of potted flowers and bonsai.

Through analysis of the relationship between monthly income level and the purchase of flowers, it can be found that, with the increase of individual monthly income level, the proportion of consumers purchasing flowers in the last year has also gradually increased. As for the reasons of no purchase, those under 3000 yuan per month hold that the price of flowers is relatively high. While, the percentage of consumers with more than 10000 yuan per month not buying flowers due to high 
price has decreased significantly.

\section{Conclusion and countermeasures}

We can find from the previous analysis that the residents of Guangzhou pay more attention to the flower information. They not only actively seek the relevant information but also hope to obtain flower maintenance knowledge through various channels. This shows that with the improvement of living standards and the importance people attach to the quality of living environment, flower consumption has become a spontaneous behavior and is becoming more and more common in Guangzhou.

According to the survey and analysis, when buying flowers, Guangzhou residents mainly buy potted flowers, fresh cut flowers, emulation flowers and bonsai. If we rank the first choice probability from big to small, then the probability to chose potted flowers firstly is $32.6 \%$, fresh cut flower $30.7 \%$, bonsai $22.3 \%$ and simulation flower $14.4 \%$. So, it can be seen that the phenomenon of "cold potted flower market but hot cut flower market" has been eliminated to a certain degree.

The factors that consumers pay attention to when buying flowers affects their purchasing decisions. In the investigation of the factors considered when buying flower products, the first is the maintenance difficulty degree of flowers. Secondly, the price and quality of flowers are considered. In addition, some consumers stress the meaning of flowers, and a small part will consider the convenience of purchase.

Why respondents choose physical stores is mainly due to the complete variety and convenience. With the popularity of network and the convenience of logistics, young people prefer to buy green plants from network, since there are more network resources and diversified selection. However, the method of ordering from Internet has not been fully accepted by residents because the quality of online shopping is sometimes not guaranteed.

The number and proportion of people buying flowers during Spring Festival rank the first, indicating that the flower festival culture has potential developing opportunities. Daily non-festival consumption is mainly for gifts to relatives and friends, stating that flowers are or are becoming media of emotional expression in the lives of Guangzhou residents.

Guangdong province is rich in non-material floral culture with various contents and forms. Flowers have been integrated into the spiritual world in social communication of residents, thus becoming the medium of transmitting friendship. The understanding level of flower culture will affect consumers' consumption of flowers. The author found in the survey that Guangzhou residents do not have a high level of understanding of flower culture. When purchasing flowers, those who "know nothing" about the meaning of flowers account for 30.7\%, those who "have a little understanding" amount to $43.4 \%$, those who "know comparatively more" account for $13.3 \%$, and those who "know very well" only occupy $12.6 \%$. In a word, the popularity of flower culture is low.

Results show that the main groups of flower consumption in Guangzhou are youth and middle-aged people. Their characteristics are higher education, stronger purchasing and consumption ability, higher aesthetic taste and higher requirements for life quality. Therefore, it is suggested to pay close attention to the actual needs of these consumer groups. In addition, it is also needed to explore the potential consumer groups by publicizing the knowledge of flowers about improving living environment, horticulture therapy, rehabilitation garden and so on, in order to explore potential consumer groups and open up a larger consumer market. The survey results reflect that most consumers lack the knowledge of flower culture, so popularizing flower science education and flower culture are particularly urgent. Public resources such as urban parks and botanical gardens can be used to highlight the cultural elements of flowers, popularize flower knowledge and increase flower culture knowledge propaganda and so on. It is also feasible to make full use of the large-scale flower exhibition and activities with flowers as the theme to run all kinds of flower markets, flower meetings and flower festivals to enrich cultural life, guiding flower consumption idea.

According to statistics, Guangzhou residents' flower consumption is mainly concentrated in holidays, and the proportion of daily household purchase is low. Moreover, the flower variety 
bought in holidays is relatively single, and is a kind of short-term consumption with a small growth space. In daily flower consumption, small potted plants are becoming more and more popular. Its main reason is the high ornamental value, low price and simple maintenance. Therefore, it is required to fully tap the variety of flower resources, introduce and improve foreign fine flower varieties to stimulate people's demand motivation. At the same time, we should increase market research, deeply analyze the consumption demands of different consumers for flower product form, color, price and purpose, through continuous and meticulous scientific research and development, in order to expand residents' flower consumption range.

According to the survey results, Guangzhou residents prefer to buy flowers in flower markets. In flower markets, there are concentrated and complete flower types, offering consumers more choices and convenience. At present, there are many large flower wholesale markets in Guangzhou, but the distribution is uneven. Therefore, it is suggested that Guangzhou flower markets should be arranged rationally and large comprehensive flower markets need to be built in every administrative district to facilitate residents. The survey also shows that more young people prefer to buy online. "Internet" sales mode is becoming a new favorite in flower market. At present, China is a country with the largest flower cultivation area, and its flower output value is also in the forefront. Therefore, the development of Chinese flower markets is of great significance to the development of the world flower market. Internet provides new opportunities for flower marketing, as well as more challenges. It is necessary to build a flower trading platform that integrates flower trade technology exchanges, exhibitions and electronic commerce. We need to fully utilize network promotion means, promote the orderly development of flower market, further excavate flower consumption potential in Guangzhou and strive to create an online shopping environment for consumers.

As the main body of flower consumption in the future, most young people do not have flower maintenance experience, but they are willing to try new things and have strong learning ability. Therefore, it is suggested to make full use of the Internet to create a characteristic marketing method of "product + service". Through the most vivid flower planting videos, it is possible to introduce planting and raising techniques, and attract consumers to buy to the maximum, thus promoting the direct growth of network marketing. At the same time, after sales, we should communicate with buyers in time, provide maintenance cultivation guidance and meet their personalized needs. Therefore, it is suggested to strengthen the network service, improve the efficiency of information feedback, give consumers timely and effective after-sales guidance, and help consumers form a satisfactory post-purchase experience.

\section{Conclusion}

The flower market is one of the representatives of rapidly growing green emerging industry, while the flower industry in China is in a situation where both opportunities and challenges coexist. Guangzhou has unique cultural geography and policy advantages for the development of flower industry.

\section{Acknowledgement}

The work was supported by the Special Funds for the Cultivation of Guangdong College Students' Scientific and Technological Innovation with the project number pdjh2017b086 and the project name Investigation on Flower Consumption of Guangzhou.

\section{References}

[1] Zhang Meng. Analysis of Factors Affecting Individual Flower Consumption in Beijing City [D]. Beijing: Beijing Forestry University, 2012.

[2] Xie Li. Investigation of Flower Industry in Beijing and Research on 2003-2015 Year Development Plan [D]. Beijing: Beijing Forestry University, 2004. 
[3] Zhao Ping. Research on the Current Situation and Prospect of Flower Industry in China [D]. Hubei: Huazhong Agricultural University, 2001.

[4] Xie Xin, Zheng Qien. Investigation Report on Main Flower Markets in Guangdong [J]. Agricultural Research and Application, 2011 (6): 59-61.

[5] Li Zhixiong. When Will the Flower Industry in China Usher the "Flower Season" [J]. Sichuan Agricultural Sciences, 2003 (3): 5-5.

[6] Chen Zhendong. Problems and Analysis of the Flower Industry in China [J]. Fujian Science\&Technology of Tropical Crops, 2006,31 (1): 34-36.

[7] Zhang Qixiang, Pan Huitang. Present Situation and Countermeasures of Introducing Foreign Ornamental Plants in China. The Science and Technology Progress of Chinese Flowers-The Second National Flower Science and Technology Information Exchange Conference [C], 2001.

[8] Yang Xiaopen. Bottlenecks and Solutions to the Flower Industry in China [J]. Productivity Research, 2006 (4): 182-183.

[9] Jiang Zehui. Speech at the 20th Anniversary Commemoration Conference of the Flower Association of China [J]. Flower Gardening in China, 2005 (5): 13-16.

[10] Yang Bo. Several Key Issues in the Development of Flower Industry in China [J]. Farm Produce Market Weekly, 2006, (19): 19. 